\title{
An Electrooculographic Method for the Evaluation of Psychotropic Drugs on Saccadic Eye Movements in Healthy Subjects
}

\author{
Raveendranadh Pilli ${ }^{1}$, Naidu M.U.R ${ }^{1}$, Usha Rani Pingali ${ }^{1} \&$ Ramesh Kumar Rao Takallapally ${ }^{1}$ \\ ${ }^{1}$ Department of Clinical Pharmacology \& Therapeutics, Nizam's Institute of Medical Sciences, Panjagutta, \\ Hyderabad, India \\ Correspondence: Raveendranadh Pilli, ICMR Advanced Centre for Clinical Pharmacodynamics, Department of \\ Clinical Pharmacology \& Therapeutics, Nizam's Institute of Medical Sciences, Panjagutta, Hyderabad, India. Tel: \\ 91-40-2335-7238. E-mail: illips@gmail.com
}

$\begin{array}{lr}\text { Received: March 18,2012 Accepted: April 5, } 2012 \quad \text { Published: June 1, } 2012 \\ \text { doi:10.5539/ijps.v4n2p75 } & \text { URL: http://dx.doi.org/10.5539/ijps.v4n2p75 }\end{array}$

\begin{abstract}
Recording of saccadic eye movements has proved to be a valuable tool for investigation of brain function and dysfunction and is very sensitive to centrally acting drugs. Since there is a lack of agreement concerning velocity-amplitude characteristics of saccadic eye movements, the present study obtained saccadic eye movements from 24 healthy subjects using indigenously developed electro-oculographic recording and analysis system in forward and countdown sequence. The validity of the system was further tested by evaluating the psychotropic effects of $5 \mathrm{mg}$ zolpidem and $500 \mathrm{mg}$ caffeine in randomized, double blind, placebo controlled three way cross over design on 24 healthy subjects. Saccadic eye movements were measured at baseline and 1, 2 and 3 hrs after administration of study medication. Mean $( \pm$ SD) values for peak saccadic velocity (PSV) over saccades of $\pm 10, \pm 20, \pm 30, \pm 40, \pm 50 \& \pm 60$ degrees were in agreement with those previously reported. Zolpidem produced statistically significant decrease in PSV compared to placebo at all the angular displacements at 1 \& 2 hrs. In contrast, caffeine produced non-significant increase in PSV at all angular displacements at $1 \mathrm{hr}$. Similarly latency period (LP) increased with zolpidem and decreased with caffeine compared to placebo. The electro-oculographic recording and analysis system is simple, sensitive to centrally acting drug effects and therefore has potential for future experimental psychomotor assessment studies.
\end{abstract}

Keywords: saccadic eye movements, electro-oculogram, peak saccadic velocity

\section{Introduction}

The human visual system is very fast and efficient at extracting information about the objects present in complex natural scenes. The speed of this visual processing can be assessed both by behavioural and neuromagnetic measurements (Kirchner \& Thrope, 2006). Eye movements seem particularly well suited for measuring processing speed because some of them can be initiated in only $80-100$ msec. These eye movements are good indicators of neurocognitive function that have been used to study the pathophysiology of schizophrenia and other neuropsychiatric disorders (Ettinger et al., 2003; Fletcher \& Sharpe, 1986).

The present article focuses on saccadic eye movements, the most common and best understood type of eye movement. Saccadic eye movements consist of two alternating events: fixations, where the eye locks on a target while the perceptual system encodes the image and saccades, where the eye moves rapidly to the next location to fixate. This alternating behavior of saccade-fixation is repeated several hundred thousand times a day and is critical for complex acts such as reading or driving an automobile. Fixation durations range from approximately $100 \mathrm{msec}$ to more than $1 \mathrm{sec}$, depending on the difficulty of the given task, and the distribution of fixation durations over particular domains is approximately exponential.

Saccades are rapid, steplike, voluntary movements of the eye that people produce as they scan a visual scene (Abrams, Meyer \& Kornblum, 1989). Saccades can be triggered by the appearance of a visual stimulus in the periphery (e.g., the sudden appearance or motion of a novel visual stimulus) or initiated voluntarily, in the absence of any overt sensory stimuli, motivated by the goals of the individual. They can also be suppressed during periods of visual fixation. Saccades have a latency of 150 to $200 \mathrm{msec}$ and once started cannot be stopped.

Peak Saccadic Velocity (PSV) appears to be a direct functional expression of changes in the level of alertness 
and is known to be sensitive to various drugs (Ball, Glue, Wilson \& Nutt, 1991). Decrease of PSV have been reported after sleep deprivation (Zils, Sprenger, Heida, Born \& Gais, 2005; Fransson et al., 2008) and administration of isoflurane (Khan et al., 1999), cyclopropane and halothane (Yoshizumi, Marshall \& Vickers, 1991) and propofol (Gao, Marshall, Sanders \& Vickers 1991) as well as benzodiazepines (Bittencourt,Wade, Smith \& Richens, 1981), where as PSV increases with verbal stimulation of subjects and after administration of d-amphetamines ( Tedeschi, Bittencourt, Smith \& Richens, 1983).

PSV is known to depend on the activity of burst neurons within the pontine reticular formation (Bittencourt et al., 1981). Saccade duration (SD) increases linearly with saccade amplitude whereas PSV increases with amplitude at a decreasing rate. The empirical main sequence relationships for horizontal saccades differ somewhat across studies because they vary across subjects, measurement techniques and analysis method, but the general pattern is always the same.

Electro-oculogram (EOG) is a representative method for the analysis of saccadic eye movement and has proven to be a readily available, relatively simple technique that provides sufficient accuracy for clinical purposes. The EOG records eye movements via a voltage difference between the cornea and retina. A prototype system for measuring saccadic eye movements has been developed, and is described in detail elsewhere (Vidapanakanti, Kakarla, Katukojwala \& Naidu, 2009). In this study we established and standardized a simple and reliable method for assessing saccadic eye movements using indigenously developed electrooculographic instrument. The effects of $5 \mathrm{mg}$ zolpidem and $500 \mathrm{mg}$ caffeine were tested versus placebo.

\section{Materials \& Methods}

\subsection{Study Participants}

Twenty four healthy participants aged 22-35 yrs, took part in the study. Following a full medical history (including smoking habits), physical examination, haematological and biochemical screening, and an electrocardiogram, volunteers were excluded if there was any evidence of physical illness or drug abuse and any abnormal screening test. Each subject was approached personally and written informed consent was taken after a full explanation of aims, procedures and risks of the study. The study was approved by the Institutional Ethics Committee and conducted in conformity with the Declaration of Helsinki. Three sets of practice eye movement tests were conducted after the medical examination and prior to the test day to introduce the subjects to the testing procedure and to make them familiar with the testing device. Data acquired in this training session were recorded, but not included in analysis. Alcohol, nicotine, chocolate or caffeine containing drinks were prohibited prior to $(12 \mathrm{~h}$ for caffeine and nicotine; $24 \mathrm{~h}$ for alcohol) and during the test day. All the recordings were carried out during the early morning between 8:00 to 10:00 AM after a light breakfast.

\subsection{Hardware of the Electroooculograph}

Figure 1 shows the configuration of a hardware system that obtains and stores images for measuring eye movement. The system consists of Visual stimulator, Saccadic stimulator, EOG Signal conditioner, Signal acquisition module (MP 30, Biopac Systems, Santa Barbara, USA) and personal computer based analysis system (BSL Pro software). The system is responsible for: Saccadic stimulus generation, EOG signal pickup and signal conditioning. A detailed description of the equipment has been given elsewhere (Vidapanakanti etal 2009).

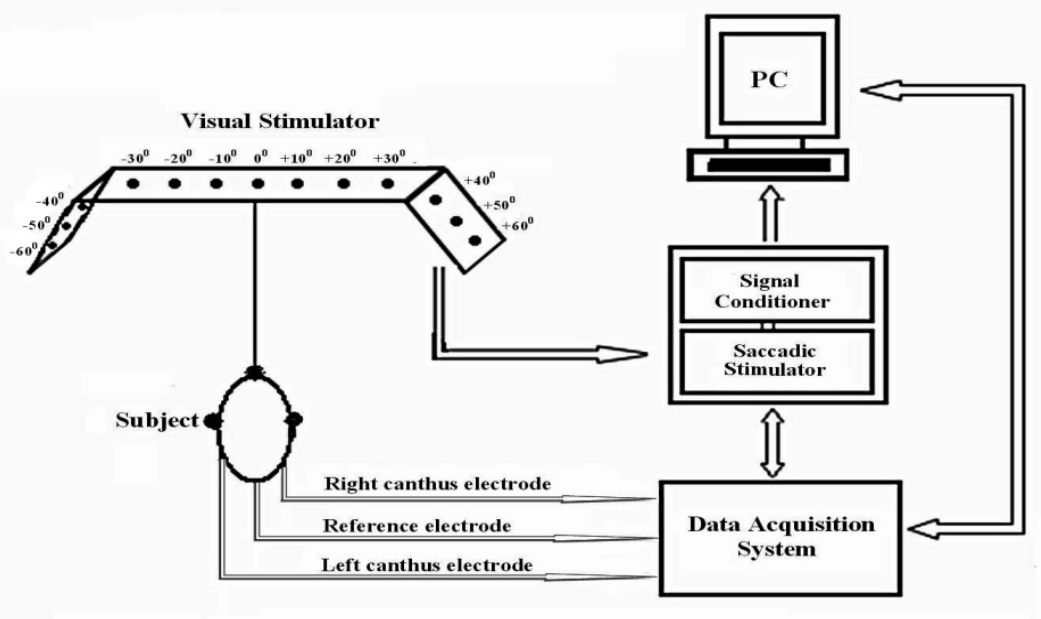

Figure 1. EOG diagnostic system 


\subsection{Procedure}

An electrooculographic method was used to measure horizontal saccadic eye movements. The recordings were obtained in a uniformly/adequately lit room, in a noise free environment with controlled temperature to avoid distraction and increase the comfort level of the subject with each subject sitting in a comfortable chair with height adjustment arrangement. Subjects sat in front of a tri-fold visual stimulator, measuring $68 \mathrm{cms}$ length and $35 \mathrm{cms}$ height on which thirteen $5-\mathrm{mm}$ red light emitting diodes (LEDs) are mounted to cover visual angles ranging from $0^{\circ}$ to $\pm 60^{\circ}$ at a distance of $40 \mathrm{cms}$. Calibration was carried out at the beginning of each trial session with subject moving his eyes to six positions on the visual stimulator. Two silver/silver chloride disposable EKG electrodes were placed $1 \mathrm{~cm}$ laterally to the outer canthus of each eye and one ground electrode below the right ear to pick up the EOG signal after the skin has been cleaned with a spirit. These are connected to a Biopac Data Acquisition system with a gain of $\times 2000$ and allowed to settle for five minutes so that the standing potentials on the electrodes are equalized. Output from the amplifier is sampled 250 times per second via an analog to digital converter, and the resulting digital information sent to an IBM-compatible personal computer for analysis. A chin rest was used to restrict the subject's head movements during the recording. The height of the subjects chair was adjusted to ensure that the subject's eyes were in visual alignment with the stimulator.

Saccades were elicited by asking the subject to watch an array of LEDs placed at a distance of $40 \mathrm{cms}$ from the glabella level with the subject's eyes, such that a movement of the eyes from one end of the display to the other end, represents 60 degrees of arc. To minimize the risk of "startle saccades", which have a different pathway of generation from anticipated saccades; subjects are asked to watch the illuminated LED as quickly as possible and not to anticipate movement of the LED stimulus. Those in which it does occur are rejected during data analysis. Trials interrupted by a blink were aborted and repeated. Instructions are verbally repeated to each subject before beginning of each trial. Initially the subject was asked to fixate on the central LED, so that the amplifier DC offset may be set to zero. The saccadic stimulus pattern was then presented and the subject was asked to look at the LED that is illuminated and move his eyes to the new position when that LED is extinguished and the next is lit. In the forward sequence (first sequence) the program causes a LED on either side of the fixation point (center LED $0^{0}$ ) to be lit alternately for $1.5 \mathrm{sec}$, starting with the LED at the center of the display (a saccade angle of $0^{0}$ ), and gradually using those nearer the center so that saccade angles of $\pm 10, \pm 20, \pm 30, \pm 40, \pm 50$ and \pm 60 degrees are elicited to and from the center LED.

During the Countdown sequence (second sequence), subjects performed the same eye movements, but from the outer extremes. The program causes a LED on either side of the fixation point (center LED $0^{0}$ ) to be lit alternately for $1.5 \mathrm{sec}$, starting with the LED at the center of the display (a saccade angle of $0^{\circ}$ ), and then the LEDs at the outer extremes of the display (a saccade angle of $60^{\circ}$ ), and gradually using those nearer the center LED so that saccade angles of $\pm 60, \pm 50, \pm 40, \pm 30, \pm 20$ and \pm 10 degrees are elicited to and from the center LED. The recorded signals were saved on computer for offline analysis using BSL Pro software. It has been found that a sequence of 12 saccades (taking about $36 \mathrm{sec}$ ) can be performed before any noticeable fatigue occurs, and on each occasion we recorded three such sequences with a brief rest of $30 \mathrm{sec}$ in between and the average of three readings was taken. After each trial, subjects received feedback regarding their responses.

\subsection{Data Analysis}

Prior to analysis, the data are smoothed using a digital filter. Each dataset was processed individually, filtered, checked for artifacts, and then its characteristics were extracted with BSL Pro software. Saccades were rejected if one of the conditions applied, namely inability to calibrate because of artifact or noise, excessively fast or slow reaction time, and a primary saccade which exceeded its allowed maximal duration or velocity. Anticipatory saccades (AS) were defined as saccades in the target direction that took the eye ahead of the target. Anticipatory saccades and express saccades with latencies smaller than $100 \mathrm{msec}$ (with respect to the go signal sstandard stimuli\}) were excluded from further analysis in all tasks. The variables of interest i.e. saccade duration (msec), PSV and latency period from target movement to saccade onset, for each saccade were extracted from the processed data (Figure 2). Saccadic duration was measured from initiation to end of saccadic eye movement. The saccade was rejected if the duration of the saccade was shorter than $30 \mathrm{msec}$, as it was regarded as measurement artifact. PSV was calculated by identifying and removing time periods where the recorded eye movements were slower than $80 \%$ and where saccades were shorter than $30 \mathrm{msec}$. If the subject made several saccades to achieve the target movement, the saccade with the highest PSV and with the largest movement distance (in degrees, usually defined as saccade amplitude) was selected. For example to calculate the PSV for amplitude of saccade of $10^{\circ}$, we took the ratio of angular displacement to its saccadic duration. The relationship between angle of eyeball displacement and measurements such as PSV is important, because it is this relationship which remains intact even when voluntary control of saccades is attempted, and is normally referred to as the main sequence. 


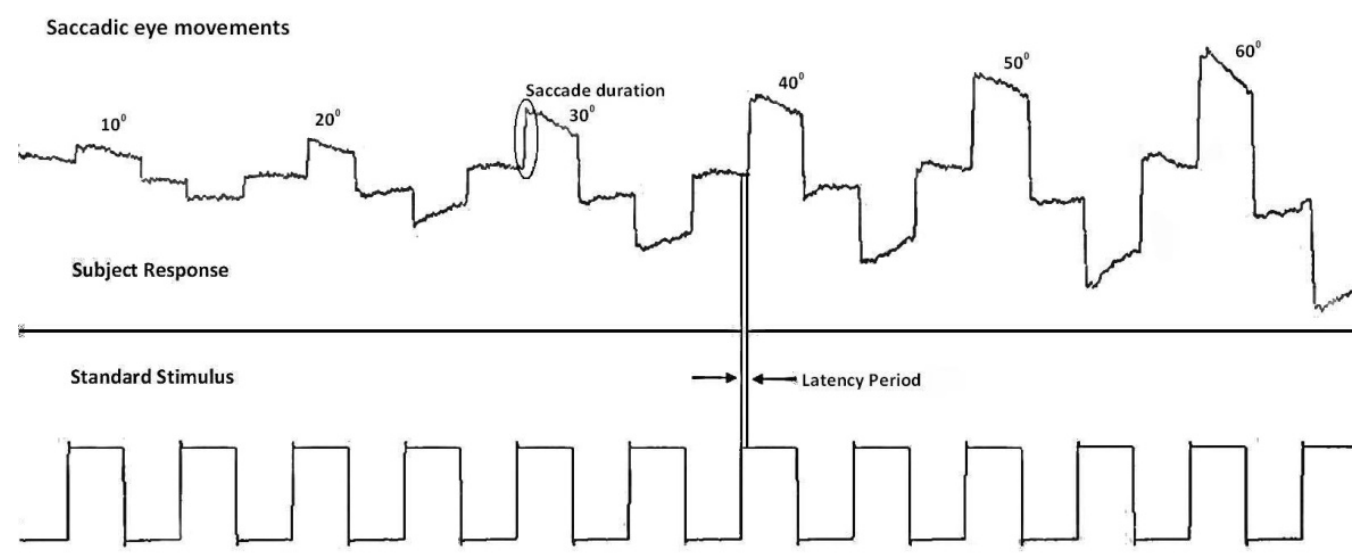

Figure 2. Signal generated by subject eye movement \& standard stimulus

In the subject response signal, the ascending signal indicates that person is looking to the right, and descending signal indicates left, midway signal corresponds to a straight look.

\subsection{Method Validation}

\section{Protocol:}

Twenty four healthy male participants aged 20-40 yrs took part in the study after they were trained on the eye movement test. The study was performed in a double blind three ways cross over design with subjects randomized to receive either a single oral dose of $5 \mathrm{mg}$ zolpidem or $500 \mathrm{mg}$ caffeine or matching placebo capsule. The treatments were separated by a washout period of one week. Subjects arrived at the laboratory following an overnight fast and abstinence of caffeine containing beverages or alcohol since $12 \mathrm{hrs}$, confirmed by a questionnaire at the beginning of the session. The procedure as described earlier for recording eye movements using forward sequence was carried out. All the recordings were carried out between 7:00 AM to 11:00 AM after a light breakfast. Saccadic eye movements were recorded before and after treatments at $1 \mathrm{hr}, 2$ hrs and 3 hrs by a single investigator. Subjects were asked to report any side effects during the study. Drug preparation and administration was done by staff members who did not participate in subject observation or data acquisition. The drug assignments for each subject were prepared by a staff member otherwise not involved in the present study using a randomization program (Statistica: Stat Soft, Inc, USA). After the study, the drug assignments were unblinded for statistical analysis. The data analysis comprised of two sessions, one in which zolpidem and placebo were compared at $0 \mathrm{hr}, 1 \mathrm{hr}, 2 \mathrm{hrs}$ and $3 \mathrm{hrs}$ and a second in which the treatments were caffeine and placebo at $0 \mathrm{hr}, 1 \mathrm{hr}$ and $3 \mathrm{hrs}$ time points.

\section{Statistical Analysis}

All statistical tests were processed using SPSS Software, Version 13 (Chicago, IL USA). Mean \pm SD values were calculated for each variable. Demographic details were summarized for all subjects using descriptive statistics. Oculomotor performance was based on the analysis of rightward and leftward saccadic eye movements. Normality of data distribution was assessed by Shapiro-Wilk test.

Correlation analyses using Spearman's rank correlation coefficient were conducted between the right eye and left eye saccadic peak velocities respectively.

ANOVA for repeated measures (averaged F) with Bonferroni correction was carried out to detect significant changes in variables over time within each session separately. Pair-wise comparisons between the two treatments (Zolpidem Vs Placebo \& Caffeine Vs Placebo) were tested for statistical significance using the paired Student's t-test. $\mathrm{P}<0.05$ was considered significant.

\section{Results}

The equipment was standardized by recording saccadic eye movements at saccade angle of $\pm 10^{0}, \pm 20^{\circ}, \pm 30^{\circ}$, $\pm 40^{\circ}, \pm 50^{\circ}$, and $\pm 60^{\circ}$ in 24 healthy subjects (male 14 and female 10). Participants were matched for age 
(27.54 $\pm 3.94 \mathrm{yrs})$ and average height, weight with a mean BMI of $21.7 \pm 3.6 \mathrm{~kg} / \mathrm{m}^{2}$. After preprocessing the recorded EOG signals, SD, PSV, LP were obtained. The results are summarized for both sequences in Table 1. The Shapiro-Wilk test of normality showed that the data was normally distributed for all parameters. As indicated in Table 1, SD and PSV increased linearly with amplitude, however latencies at various amplitudes were not significantly different. No significant difference was observed for any saccade parameter between males and females participant. Correlation of right and left peak saccadic velocities in forward sequence demonstrated non significant positive correlation at saccadic angles $10^{\circ}, 20^{\circ}, 40^{\circ}, 50^{\circ}$. However at $30^{\circ} \& 60^{\circ}$ non significant negative correlation was observed. Where as in the countdown sequence, right and left peak saccadic velocities were positively correlated at all saccadic angles though there was no significance.

Table $1 \&$ Figure 3 show that the rightward saccades were modestly slower than leftward saccades in forward sequence. Similarly latency period of right ward saccades was slower than leftward saccades. A similar effect was observed between rightward and leftward saccades in countdown sequence.

Table 1. Rightward \& Leftward saccadic eye movements in healthy subjects

\begin{tabular}{ccccccc}
\hline \multicolumn{3}{c}{ Rightward Movements } & \multicolumn{3}{c}{ Leftward Movements } \\
$\begin{array}{c}\text { Angular } \\
\text { Displacement }\end{array}$ & $\begin{array}{c}\text { SD } \\
(\mathrm{msec})\end{array}$ & $\begin{array}{c}\text { PSV } \\
(\% / \mathrm{sec})\end{array}$ & $\begin{array}{c}\text { LP } \\
(\mathrm{msec})\end{array}$ & $\begin{array}{c}\text { SD } \\
(\mathrm{msec})\end{array}$ & $\begin{array}{c}\text { PSV } \\
(\% / \mathrm{sec})\end{array}$ & $\begin{array}{c}\text { LP } \\
(\mathrm{msec})\end{array}$ \\
\hline \multicolumn{2}{c}{ Forward Sequence } & & & & & \\
$10^{0}$ & $42.13 \pm 5.94$ & $241.7 \pm 33.02$ & $172.2 \pm 19.58$ & $46.63 \pm 4.04$ & $216 \pm 18.48$ & $164.7 \pm 22.17$ \\
$20^{\circ}$ & $54.16 \pm 1.85$ & $369.7 \pm 12.83$ & $178.1 \pm 26.02$ & $59.56 \pm 4.08$ & $337.2 \pm 22.35$ & $162.5 \pm 22.80$ \\
$30^{\circ}$ & $70.41 \pm 2.78$ & $426.7 \pm 16.63$ & $176.3 \pm 24.87$ & $71.94 \pm 5.03$ & $418.9 \pm 28.81$ & $165 \pm 26.08$ \\
$40^{0}$ & $84.81 \pm 3.67$ & $472.5 \pm 20.4$ & $182.8 \pm 28.98$ & $88.63 \pm 6.20$ & $453.5 \pm 32.48$ & $174.8 \pm 34.48$ \\
$50^{\circ}$ & $99.5 \pm 4.70$ & $503.5 \pm 23.01$ & $192.5 \pm 32.94$ & $103.3 \pm 9.65$ & $487.9 \pm 45.26$ & $184.7 \pm 27.72$ \\
$60^{\circ}$ & $113.6 \pm 6.38$ & $529.8 \pm 30.11$ & $202.5 \pm 33.36$ & $120.8 \pm 8.59$ & $499.3 \pm 36.67$ & $196.6 \pm 22.91$ \\
Countdown Sequence & & & & & \\
$10^{\circ}$ & $36.97 \pm 4.26$ & $274.6 \pm 36.34$ & $181.7 \pm 29.17$ & $43.25 \pm 4.26$ & $233.3 \pm 23.1$ & $156.3 \pm 22.99$ \\
$20^{\circ}$ & $48.41 \pm 4.34$ & $414.5 \pm 36.18$ & $186.7 \pm 36.64$ & $55.94 \pm 5.34$ & $360.6 \pm 33.85$ & $169.7 \pm 30.68$ \\
$30^{\circ}$ & $61.66 \pm 4.35$ & $487.4 \pm 34.91$ & $186.4 \pm 22.2$ & $71.81 \pm 5.23$ & $419.9 \pm 31.96$ & $178.4 \pm 26.88$ \\
$40^{\circ}$ & $76 \pm 5.09$ & $530.6 \pm 35.01$ & $194 \pm 18.09$ & $79.81 \pm 6.86$ & $504.5 \pm 42.04$ & $182.9 \pm 37.81$ \\
$50^{\circ}$ & $89.84 \pm 4.86$ & $556.4 \pm 31.3$ & $213.3 \pm 39.45$ & $100.9 \pm 7.49$ & $498.1 \pm 35.31$ & $185.9 \pm 36.02$ \\
$60^{\circ}$ & $102.1 \pm 7.45$ & $588.4 \pm 44.04$ & $246.9 \pm 32.73$ & $115.6 \pm 10.14$ & $522.4 \pm 42.84$ & $209.1 \pm 34.65$ \\
\hline
\end{tabular}

Data represented as Mean $\pm \mathrm{SD}$ 

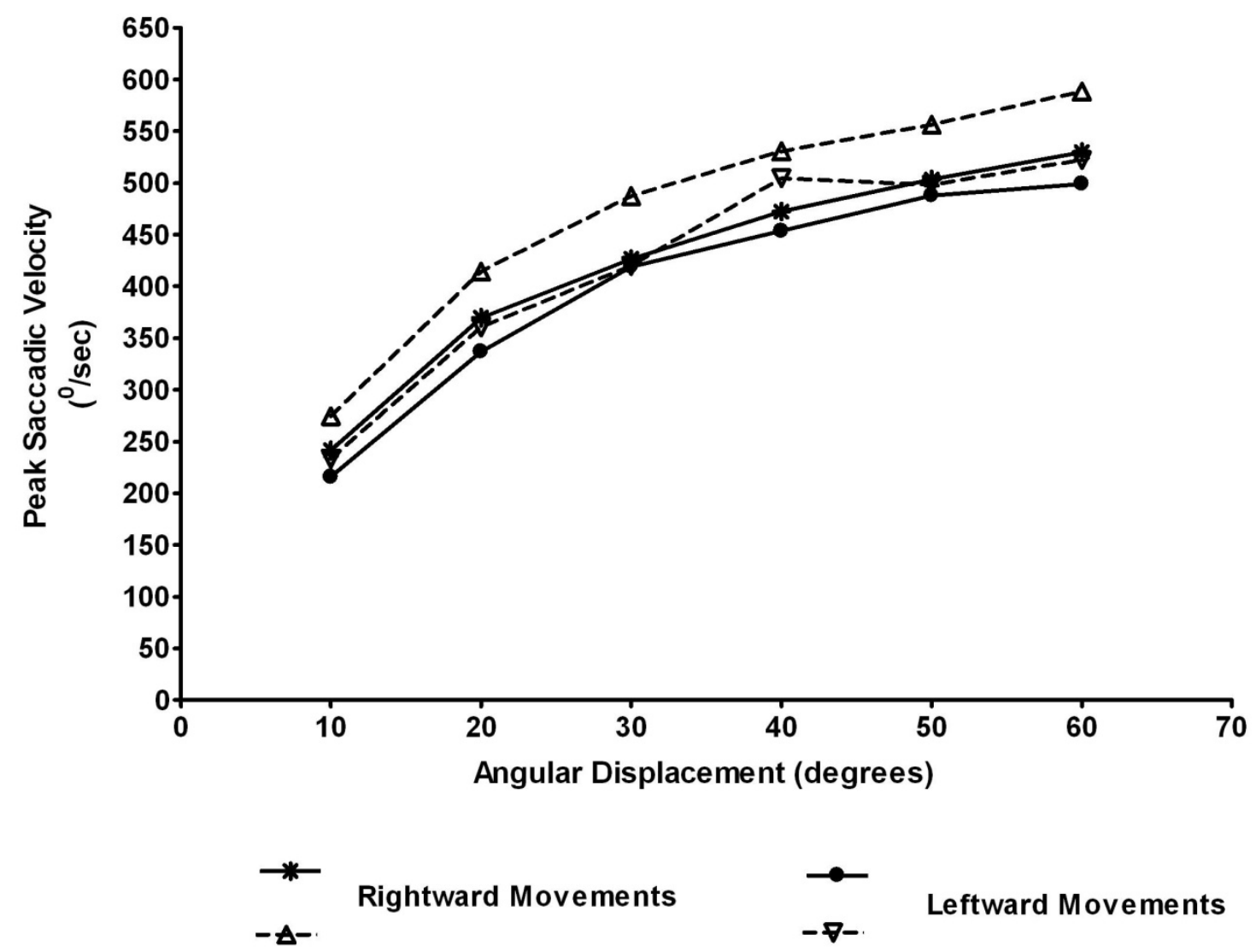

Figure 3. Peak saccadic velocity of forward \& countdown sequence;

Straight lines \& dotted lines represent forward \& countdown sequence

\subsection{Validation Results}

To confirm the validity of the above method, we have used zolpidem and caffeine as reference study medication in 24 healthy male individuals. Two subjects due to dizziness and vomiting in zolpidem group and two subjects from cafeine group due to gastric irritation were excluded. So the data of 20 subjects were analyzed, of which participants were of average height and weight for their age (29.75 \pm 3.98 yrs) and their mean BMI was $22.4 \pm 2.6$ $\mathrm{kg} / \mathrm{m}^{2}$.

There were no significant differences in baseline characteristics between the three sessions (Table 2, $3 \& 4$ ). 
Table 2. Saccadic eye movements before and after oral intake of Zolpidem

\begin{tabular}{|c|c|c|c|c|c|c|c|}
\hline \multirow{3}{*}{ Time } & \multirow{3}{*}{$\begin{array}{c}\text { Angular } \\
\text { Displacement }\end{array}$} & \multicolumn{3}{|c|}{ Rightward movements } & \multicolumn{3}{|c|}{ Leftward Movements } \\
\hline & & SD & PSV & $\mathrm{LP}$ & $\mathrm{SD}$ & PSV & LP \\
\hline & & $(\mathrm{msec})$ & $(\% / \mathrm{sec})$ & $(\mathrm{msec})$ & $(\mathrm{msec})$ & $(\% / \mathrm{sec})$ & $(\mathrm{msec})$ \\
\hline \multirow[t]{6}{*}{$0 \mathrm{hr}$} & $10^{\circ}$ & $43.91 \pm 4.64$ & $234.8 \pm 19.29$ & $166.9 \pm 22.84$ & $48.41 \pm 4.76$ & $208.5 \pm 20.79$ & $165.4 \pm 19.97$ \\
\hline & $20^{\circ}$ & $55.09 \pm 4.72$ & $365.6 \pm 32.14$ & $170.1 \pm 33.15$ & $61.69 \pm 3.94$ & $325.5 \pm 20.9$ & $158.6 \pm 29.55$ \\
\hline & $30^{\circ}$ & $73.75 \pm 4.16$ & $409 \pm 24.13$ & $168.6 \pm 23.15$ & $72.94 \pm 4.13$ & $412.6 \pm 23.6$ & $167.4 \pm 29.72$ \\
\hline & $40^{\circ}$ & $87.94 \pm 6.53$ & $460.3 \pm 30.51$ & $168.7 \pm 32.16$ & $88.63 \pm 7.33$ & $454.3 \pm 37.83$ & $166.8 \pm 24.13$ \\
\hline & $50^{\circ}$ & $101.5 \pm 10.11$ & $497.5 \pm 50.19$ & $179.8 \pm 52.08$ & $104.4 \pm 9.44$ & $482.4 \pm 41.19$ & $173.9 \pm 22.51$ \\
\hline & $60^{\circ}$ & $115.9 \pm 9.46$ & $523 \pm 42.54$ & $193.9 \pm 49.51$ & $120.5 \pm 8.82$ & $500.4 \pm 36.65$ & $176.2 \pm 39.72$ \\
\hline \multirow[t]{6}{*}{$1 \mathrm{hr}$} & $10^{0}$ & $48.31 \pm 7.60$ & $215.9 \pm 38.35$ & 212.69 .21 & $58.56 \pm 6.95$ & $172.9 \pm 19.59$ & $182.1 \pm 38.79$ \\
\hline & $20^{0}$ & $66.59 \pm 9.37$ & $307.7 \pm 39.67$ & $210.6 \pm 60.16$ & $69.69 \pm 4.30$ & $288 \pm 17.57$ & $186.1 \pm 50.42$ \\
\hline & $30^{\circ}$ & $85.34 \pm 10.14$ & $360 \pm 46.04$ & $195 \pm 50.93$ & $85.72 \pm 8.19$ & $352.9 \pm 32.57$ & $185.9 \pm 35.16$ \\
\hline & $40^{\circ}$ & $102.6 \pm 12.83$ & $396.6 \pm 46.43$ & $192.3 \pm 55.23$ & $99.16 \pm 9.75$ & $407.2 \pm 40.99$ & $181.7 \pm 40.33$ \\
\hline & $50^{\circ}$ & $121.6 \pm 15.43$ & $420.7 \pm 53.42$ & $203.9 \pm 49.29$ & $123.8 \pm 15.6$ & $410 \pm 52.49$ & $186.8 \pm 28.51$ \\
\hline & $60^{0}$ & $143.9 \pm 17.37$ & $423.6 \pm 48.66$ & $197.2 \pm 53.8$ & $141.8 \pm 11.2$ & $425.9 \pm 36.92$ & $188.2 \pm 47.18$ \\
\hline \multirow[t]{6}{*}{$2 \mathrm{hrs}$} & $10^{0}$ & $50.88 \pm 10.05$ & $211.5 \pm 39.08$ & $202.2 \pm 53.95$ & $58.63 \pm 4.89$ & $171.7 \pm 13.96$ & $176.3 \pm 27.96$ \\
\hline & $20^{\circ}$ & $67.94 \pm 9.35$ & $302.5 \pm 42.79$ & $197.3 \pm 42.4$ & $74.47 \pm 6.67$ & $270.7 \pm 25.46$ & $182.4 \pm 16.24$ \\
\hline & $30^{\circ}$ & $84.06 \pm 9.61$ & $211.5 \pm 39.08$ & $203.6 \pm 34.6$ & $81.13 \pm 7.53$ & $372.9 \pm 35.84$ & $176.8 \pm 30.77$ \\
\hline & $40^{\circ}$ & $97.66 \pm 10.47$ & $417.6 \pm 45.42$ & $196.4 \pm 30.22$ & $98.69 \pm 7.80$ & $407.7 \pm 31.52$ & $183.1 \pm 42.96$ \\
\hline & $50^{\circ}$ & $123.4 \pm 14.09$ & $412.3 \pm 45.54$ & $211.1 \pm 49.91$ & $122.9 \pm 10.3$ & $409.6 \pm 35.28$ & $179.5 \pm 36.08$ \\
\hline & $60^{\circ}$ & $142.4 \pm 20.07$ & $435.2 \pm 65.4$ & $214.7 \pm 42.11$ & $148.3 \pm 15.1$ & $408.9 \pm 43.94$ & $188.8 \pm 43.75$ \\
\hline \multirow[t]{6}{*}{$3 \mathrm{hrs}$} & $10^{0}$ & $46.88 \pm 5.88$ & $219.4 \pm 25.88$ & $173.8 \pm 19.68$ & $55.63 \pm 4.19$ & $180.3 \pm 13.54$ & $174.6 \pm 23.43$ \\
\hline & $20^{\circ}$ & $64.06 \pm 7.35$ & $319.5 \pm 34.67$ & $190.3 \pm 39.22$ & $68.91 \pm 6.98$ & $293.3 \pm 30.74$ & $166.3 \pm 23.93$ \\
\hline & $30^{\circ}$ & $77.19 \pm 8.41$ & $397.5 \pm 48.64$ & $174.5 \pm 25.34$ & $81.16 \pm 6.21$ & $372.9 \pm 28.32$ & $173.8 \pm 31$ \\
\hline & $40^{\circ}$ & $97.19 \pm 19.19$ & $427.7 \pm 65.45$ & $186.7 \pm 37.75$ & $95.84 \pm 10.9$ & $425.4 \pm 46.61$ & $162.3 \pm 25.06$ \\
\hline & $50^{\circ}$ & $120.9 \pm 16.01$ & $422.8 \pm 52.92$ & $193 \pm 59.49$ & $117.7 \pm 6.47$ & $426.5 \pm 23.8$ & $167.7 \pm 24.19$ \\
\hline & $60^{\circ}$ & $146.7 \pm 22.71$ & $419.5 \pm 62.74$ & $198 \pm 63$ & $142.3 \pm 10.7$ & $420.6 \pm 33.82$ & $173.2 \pm 39.61$ \\
\hline
\end{tabular}

Data represented as Mean $\pm \mathrm{SD}$ 
Table 3. Saccadic eye movements before and after oral intake of Placebo

\begin{tabular}{|c|c|c|c|c|c|c|c|}
\hline \multirow{3}{*}{ Time } & \multirow{3}{*}{$\begin{array}{c}\text { Angular } \\
\text { Displacement }\end{array}$} & \multicolumn{3}{|c|}{ Rightward movements } & \multicolumn{3}{|c|}{ Leftward Movements } \\
\hline & & SD & PSV & LP & $\mathrm{SD}$ & PSV & LP \\
\hline & & $(\mathrm{msec})$ & $(\% / \mathrm{sec})$ & $(\mathrm{msec})$ & $(\mathrm{msec})$ & $(\% / \mathrm{sec})$ & $(\mathrm{msec})$ \\
\hline \multirow[t]{6}{*}{$0 \mathrm{hr}$} & $10^{0}$ & $44.21 \pm 3.52$ & $227.5 \pm 17.84$ & $165 \pm 20.13$ & $46.54 \pm 2.24$ & $215.3 \pm 10.41$ & $155.5 \pm 11.94$ \\
\hline & $20^{\circ}$ & $55.42 \pm 2.14$ & $361.4 \pm 14.15$ & $174.1 \pm 21.72$ & $56.67 \pm 3.74$ & $354.4 \pm 23.94$ & $152.2 \pm 21.02$ \\
\hline & $30^{0}$ & $71.96 \pm 4.74$ & $416.7 \pm 21.85$ & $172.6 \pm 15.76$ & $72.75 \pm 5.29$ & $414.5 \pm 31.78$ & $151.3 \pm 14.58$ \\
\hline & $40^{\circ}$ & $86.38 \pm 9.17$ & $468.9 \pm 44.67$ & $171.7 \pm 25.03$ & $86.29 \pm 3.39$ & $464.2 \pm 18.46$ & $160 \pm 20.97$ \\
\hline & $50^{\circ}$ & $102.1 \pm 6.38$ & $491.6 \pm 30.82$ & $183.9 \pm 17.07$ & $99.08 \pm 8.90$ & $508.3 \pm 44.1$ & $167.1 \pm 26.35$ \\
\hline & $60^{\circ}$ & $119.1 \pm 11.8$ & $513.8 \pm 54.17$ & $214.5 \pm 16.9$ & $116.3 \pm 9.56$ & $518.9 \pm 41.62$ & $181 \pm 12.8$ \\
\hline \multirow[t]{6}{*}{$1 \mathrm{hr}$} & $10^{0}$ & $41.96 \pm 3.23$ & $239.6 \pm 18.39$ & $170.9 \pm 18.26$ & $45.83 \pm 4.99$ & $220.5 \pm 23.52$ & $158.6 \pm 14.16$ \\
\hline & $20^{0}$ & $56.46 \pm 7.10$ & $363.1 \pm 45.39$ & $168.8 \pm 20$ & $58.5 \pm 3.39$ & $343 \pm 20.21$ & $156.9 \pm 22.13$ \\
\hline & $30^{\circ}$ & $76.08 \pm 7.55$ & $404.3 \pm 42.65$ & $169.1 \pm 25.96$ & $73.71 \pm 6.45$ & $409.8 \pm 35.16$ & $161.3 \pm 19.54$ \\
\hline & $40^{\circ}$ & $86.25 \pm 9.91$ & $471.1 \pm 49.88$ & $181.9 \pm 21.64$ & $86.96 \pm 4.84$ & $461.2 \pm 24.60$ & $177.1 \pm 24.4$ \\
\hline & $50^{\circ}$ & $102.9 \pm 12.91$ & $495.4 \pm 55.42$ & $184.7 \pm 23.3$ & $99.96 \pm 7.45$ & $502.7 \pm 36.71$ & $163.5 \pm 24.53$ \\
\hline & $60^{\circ}$ & $120.3 \pm 12.16$ & $512.3 \pm 55.44$ & $218.4 \pm 18.99$ & $115.7 \pm 6.98$ & $520.5 \pm 31.35$ & $183.5 \pm 25.71$ \\
\hline \multirow[t]{6}{*}{2 hrs } & $10^{0}$ & $42.58 \pm 2.66$ & $239.4 \pm 14.3$ & $167.5 \pm 16.79$ & $46.42 \pm 5.29$ & $218.1 \pm 25.44$ & $164.3 \pm 18.48$ \\
\hline & $20^{0}$ & $56.71 \pm 5.11$ & $358.8 \pm 31.28$ & $174.3 \pm 24.85$ & $57.42 \pm 3.11$ & $349.3 \pm 19.01$ & $156.2 \pm 10.23$ \\
\hline & $30^{\circ}$ & $72.79 \pm 7.45$ & $418.6 \pm 39.8$ & $170.8 \pm 29.75$ & $72.79 \pm 4.19$ & $413.4 \pm 23.69$ & $165 \pm 21.71$ \\
\hline & $40^{\circ}$ & $86.29 \pm 6.90$ & $473.1 \pm 41.79$ & $181.8 \pm 22.67$ & $87.08 \pm 7.88$ & $462.7 \pm 40.97$ & $158.4 \pm 16.35$ \\
\hline & $50^{\circ}$ & $104.3 \pm 12.4$ & $483.5 \pm 48$ & $183.1 \pm 16.59$ & $101.7 \pm 10.23$ & $496.1 \pm 46.43$ & $165.1 \pm 21.39$ \\
\hline & $60^{\circ}$ & $119 \pm 9.77$ & $497.2 \pm 48.18$ & $211.6 \pm 16.97$ & $116.6 \pm 8.77$ & $517.3 \pm 38.31$ & $177.2 \pm 20.78$ \\
\hline \multirow[t]{6}{*}{$3 \mathrm{hrs}$} & $10^{0}$ & $43.92 \pm 4.47$ & $233.4 \pm 22.09$ & $178.5 \pm 21.67$ & $47.58 \pm 5.02$ & $212.5 \pm 24.14$ & $156 \pm 18.95$ \\
\hline & $20^{\circ}$ & $54.58 \pm 5.41$ & $374.4 \pm 32.55$ & $183.5 \pm 21.51$ & $58.04 \pm 3.61$ & $345.8 \pm 21.36$ & $172.2 \pm 24.34$ \\
\hline & $30^{0}$ & $74.29 \pm 6.18$ & $407.7 \pm 32.63$ & $177.1 \pm 23.09$ & $71.96 \pm 6.09$ & $419.6 \pm 35.07$ & $166.1 \pm 21.78$ \\
\hline & $40^{\circ}$ & $86.92 \pm 4.93$ & $463.4 \pm 26.49$ & $179.1 \pm 30.46$ & $87.54 \pm 3.19$ & $457.5 \pm 16.7$ & $166.5 \pm 21.53$ \\
\hline & $50^{\circ}$ & $100.3 \pm 11.21$ & $502.2 \pm 50.33$ & $181.9 \pm 17.62$ & $99.29 \pm 7.84$ & $506.4 \pm 39.82$ & $169.2 \pm 13.37$ \\
\hline & $60^{\circ}$ & $122.3 \pm 13.44$ & $500.5 \pm 52.26$ & $209.2 \pm 25.71$ & $117.3 \pm 9.37$ & $514.4 \pm 41.08$ & $177 \pm 20.37$ \\
\hline
\end{tabular}

Data represented as Mean $\pm \mathrm{SD}$ 
Table 4. Saccadic eye movements before and after oral intake of Caffeine

\begin{tabular}{|c|c|c|c|c|c|c|c|}
\hline \multirow[b]{2}{*}{ Time } & \multirow{3}{*}{$\begin{array}{c}\text { Angular } \\
\text { Displacement }\end{array}$} & \multicolumn{3}{|c|}{ Rightward movements } & \multicolumn{3}{|c|}{ Leftward Movements } \\
\hline & & SD & PSV & LP & SD & PSV & LP \\
\hline & & $(\mathrm{msec})$ & $(\% / \mathrm{sec})$ & $(\mathrm{msec})$ & $(\mathrm{msec})$ & $(0 / \mathrm{sec})$ & $(\mathrm{msec})$ \\
\hline \multirow[t]{6}{*}{$\mathbf{0 ~ h r}$} & $10^{0}$ & $49.23 \pm 6.4$ & $223.4 \pm 19.82$ & $164.8 \pm 28.29$ & $46.63 \pm 4.29$ & $216.1 \pm 19.86$ & $149.7 \pm 19.15$ \\
\hline & $20^{\circ}$ & $54 \pm 6.25$ & $378.8 \pm 48.38$ & $171.8 \pm 20.1$ & $58.07 \pm 3.63$ & $345.7 \pm 21.95$ & $163.7 \pm 18.01$ \\
\hline & $30^{\circ}$ & $72.57 \pm 6.14$ & $418.2 \pm 32.3$ & $174.8 \pm 18.04$ & $73.4 \pm 7.24$ & $412.7 \pm 43.07$ & $155.3 \pm 19.6$ \\
\hline & $40^{\circ}$ & $86.13 \pm 8.97$ & $470.6 \pm 45.59$ & $172.3 \pm 26.48$ & $90.33 \pm 6.11$ & $444.7 \pm 29.39$ & $162.3 \pm 22.75$ \\
\hline & $50^{\circ}$ & $101.5 \pm 9.22$ & $499.8 \pm 41.26$ & $183.3 \pm 19.23$ & $104 \pm 7.41$ & $483.10 \pm 34.60$ & $167.1 \pm 18.16$ \\
\hline & $60^{\circ}$ & $117.8 \pm 10.52$ & $516.4 \pm 47.1$ & $207.7 \pm 16.76$ & $122.9 \pm 7.91$ & $490 \pm 32.82$ & $175 \pm 19.73$ \\
\hline \multirow[t]{6}{*}{$1 \mathrm{hr}$} & $10^{0}$ & $45.3 \pm 9.26$ & $234.7 \pm 49.06$ & $170 \pm 25.34$ & $44.33 \pm 4.25$ & $227.6 \pm 22.8$ & $170 \pm 25.34$ \\
\hline & $20^{0}$ & $50.27 \pm 6.91$ & $405.8 \pm 62.78$ & $163.3 \pm 29.86$ & $55.8 \pm 6.42$ & $363.2 \pm 44.81$ & $163.3 \pm 29.36$ \\
\hline & $30^{\circ}$ & $66.9 \pm 6.47$ & $452.5 \pm 45.5$ & $171.2 \pm 27.96$ & $69.97 \pm 5.54$ & $431.1 \pm 35.67$ & $171.2 \pm 27.96$ \\
\hline & $40^{\circ}$ & $80.73 \pm 8.05$ & $502.1 \pm 49.78$ & $153.2 \pm 27.93$ & $85.07 \pm 7.40$ & $473.5 \pm 40.05$ & $153.2 \pm 27.93$ \\
\hline & $50^{0}$ & $93.23 \pm 6.85$ & $543.1 \pm 31.94$ & $165.5 \pm 19.58$ & $97.23 \pm 5.04$ & $515.5 \pm 26.67$ & $165.5 \pm 19.58$ \\
\hline & $60^{\circ}$ & $110.1 \pm 8.36$ & $559.7 \pm 40.46$ & $176.2 \pm 29.27$ & $115.7 \pm 7.16$ & $520.3 \pm 31.78$ & $176.2 \pm 29.27$ \\
\hline \multirow[t]{6}{*}{$3 \mathrm{hrs}$} & $10^{0}$ & $44.87 \pm 2.84$ & $229.1 \pm 23.7$ & $166 \pm 21.3$ & $48.33 \pm 6.20$ & $213.8 \pm 27.4$ & $154.3 \pm 19.2$ \\
\hline & $20^{0}$ & $60.77 \pm 4.37$ & $342.3 \pm 30.4$ & $182 \pm 41.2$ & $57.3 \pm 2.15$ & $350.6 \pm 12.4$ & $163.3 \pm 18.7$ \\
\hline & $30^{\circ}$ & $69.4 \pm 5.66$ & $436.5 \pm 32.6$ & $175.2 \pm 20.2$ & $71.67 \pm 4.75$ & $423.1 \pm 27.0$ & $168.8 \pm 28.0$ \\
\hline & $40^{0}$ & $83.1 \pm 7.53$ & $487.7 \pm 43.9$ & $176.5 \pm 21.6$ & $88.4 \pm 9.17$ & $461.6 \pm 46.5$ & $157.6 \pm 25.3$ \\
\hline & $50^{\circ}$ & $100.5 \pm 8.40$ & $501.2 \pm 40.5$ & $177.2 \pm 35.6$ & $104 \pm 11.25$ & $493.5 \pm 49.1$ & $169.8 \pm 19.8$ \\
\hline & $60^{\circ}$ & $117.1 \pm 13.66$ & $519.7 \pm 56.6$ & $188.3 \pm 35.9$ & $120.6 \pm 7.70$ & $502.8 \pm 31.3$ & $170 \pm 25.3$ \\
\hline
\end{tabular}

Data represented as Mean $\pm \mathrm{SD}$ 


\subsubsection{Effect of Zolpidem on Saccadic Eye Movements}

Results for the outcome measures are depicted in Table $2 \& 3$.

The results of the saccadic eye movement tests showed that zolpidem significantly increased SD, lowered PSV and LP compared to placebo for both rightward and leftward eye movements.

\section{Saccadic duration:}

ANOVA with repeated measures detected significant treatment effect on saccadic duration for rightward movements at $10^{\circ}(p=0.012), 20^{\circ}(p=0.001), 30^{\circ}(p=0.001), 40^{\circ}(p=0.002), 50^{\circ}(p<0.0001), 60^{\circ}(p<$ $0.0001)$. A significant effect was also observed for leftward movements at $10^{\circ}(p=0.001), 20^{\circ}(p<0.0001), 30^{\circ}$ $(p=0.001), 40^{\circ}(p=0.001), 50^{\circ}(p<0.0001), 60^{\circ}(p<0.0001)$.

Compared to baseline a significant effect was observed for rightward movements at $1 \mathrm{hr}$ and $2 \mathrm{hrs}$ at $20^{\circ}, 30^{\circ}$, $40^{\circ}, 50^{\circ}, 60^{\circ}$ No significant effect was noticed at $3 \mathrm{hrs}$ at $20^{\circ}, 30^{\circ}, 40^{\circ}$, where as at $50^{\circ}$ and $60^{\circ}$ showed significant effect at $3 \mathrm{hrs}$ also. A significant effect is also observed for leftward movements at $1 \mathrm{hr}, 2 \mathrm{hrs}$, and $3 \mathrm{hrs}$ for all amplitudes.

Specific time points at which significant zolpidem versus placebo differences occurred were identified by two-tailed paired t-tests. Saccadic duration differed at $1 \mathrm{hr}$ at $10^{\circ}(p=0.001), 20^{\circ}(p=0.02), 30^{\circ}(p=0.02)$, $\left.40^{\circ}(p=0.01), 50^{\circ}(p=0.007), 60^{\circ}(p=0.004)\right\}, 2$ hrs at $10^{\circ}(p=0.01), 20^{\circ}(p=0.001), 30^{\circ}(p=0.01), 40^{\circ}(p=0.02)$, $\left.50^{\circ}(p=0.004), 60^{\circ}(p=0.009)\right\}$ and $3 \mathrm{hrs}\left\{\right.$ at $\left.50^{\circ}(p=0.01), 60^{\circ}(p=0.04)\right\}$ for rightward movements.

In case of leftward eye movements, saccadic duration differed at $1 \mathrm{hr}$ at $10^{0}(p=0.004), 20^{0}(p<0.0001)$, $\left.30^{0}(p=0.008), 40^{0}(p=0.01), 50^{0}(p<0.0001), 60^{0}(p<0.0001)\right\}, 2$ hrs at $10^{0} \quad(\mathrm{p}=0.001), 20^{0}(p=0.001)$, $\left.30^{\circ}(p=0.002), 40^{\circ}(p=0.002), 50^{\circ}(p<0.0001), 60^{\circ}(p=0.001)\right\}$ and $3 \mathrm{hrs}$ at $20^{\circ}(p=0.007), 30^{\circ}(p=0.008)$, $\left.40^{\circ}(p=0.02), 50^{\circ}(p<0.0001), 60^{\circ}(p=0.002)\right\}$.

\section{Peak saccadic velocity:}

A significant reduction of PSV was observed with Zolpidem for rightward movements at $10^{\circ}(p=0.04), 20^{0}(p=$ $0.001), 30^{\circ}(p<0.0001), 40^{\circ}(p<0.0001), 50^{\circ}(p<0.0001), 60^{\circ}(p<0.0001)$. A significant effect was also observed for leftward movements at $10^{0}(p=0.001), 20^{\circ}(p<0.0001), 30^{\circ}(p=0.001), 40^{\circ}(p=0.001), 50^{\circ}(p=$ $0.001), 60^{0}(p=0.001)$.

Compared to baseline a significant decrease in PSV can be observed for rightward movements at $1 \mathrm{hr}$ and $2 \mathrm{hrs}$ at $20^{\circ}, 30^{\circ}, 40^{\circ}, 50^{\circ}, 60^{\circ}$ No significant effect can be seen at $3 \mathrm{hrs}$ at $20^{\circ}, 30^{\circ}, 40^{\circ}$, where as $50^{\circ}$ and $60^{\circ}$ showed significant effect at $3 \mathrm{hrs}$ also. A similar significant effect is also observed for leftward movements at $1 \mathrm{hr}, 2 \mathrm{hrs,}$ and $3 \mathrm{hrs}$ for all amplitudes except at $40^{\circ}$ at $3 \mathrm{hrs}$.

Specific time points at which significant zolpidem versus placebo differences occurred were identified by two-tailed t-test. PSV decreased at $1 \mathrm{hr}\left\{\right.$ at $10^{\circ}(p=0.001), 20^{\circ}(p=0.01), 30^{\circ}(p=0.01), 40^{\circ}(p=0.01)$, $\left.50^{0}(p=0.008), 60^{0}(p=0.002)\right\}, 2 \mathrm{hrs}\left\{\right.$ at $10^{0}(p=0.01), 20^{0}(p=0.002), 30^{0}(p=0.002), 40^{0}(p=0.02), 50^{\circ}(p=0.002)$, $\left.60^{\circ}(p=0.001)\right\}$ and $3 \mathrm{hrs}\left\{\right.$ at $\left.50^{\circ}(p=0.01), 60^{\circ}(p=0.04)\right\}$ for rightward movements.

In case of leftward eye movements PSV decreased at $1 \mathrm{hr}$ at $10^{0}(p=0.003), 20^{0}(p<0.0001), 30^{0}(p=0.007)$, $\left.40^{\circ}(p=0.001), 50^{\circ}(p<0.0001), 60^{\circ}(p<0.0001)\right\}, 2$ hrs at $10^{0}(p=0.001), 20^{0} \quad(p<0.0001), 30^{\circ}(p=0.002)$, $\left.40^{\circ}(p=0.002), 50^{\circ}(p<0.0001), 60^{\circ}(p<0.0001)\right\}$ and $3 \mathrm{hrs}$ at $10^{\circ}(p<0.0001), 20^{\circ}(p=0.007), 30^{\circ}(p=0.01)$, $\left.40^{\circ}(p=0.03), 50^{\circ}(p<0.0001), 60^{\circ}(p=0.001)\right\}$.

\section{Latency period:}

ANOVA with repeated measures showed a significant decrease in latency period for rightward movements at $10^{0}$ $(p<0.0001), 20^{\circ}(p=0.009), 30^{\circ}(p=0.007)$ only. Where as in case of leftward movements a significant effect was observed at $20^{\circ}(p=0.01)$

Compared to baseline latency period increased at all saccadic amplitude at $1 \mathrm{hr}, 2 \mathrm{hrs}$ and $3 \mathrm{hrs}$, however a significant effect can be only seen at $10^{\circ}$ and $20^{\circ}$ at 2 hrs only for rightward movements. In case of leftward movements, though there was an increase in the latency period at all saccadic amplitudes, the effect was not significant.

Specific time points at which significant zolpidem versus placebo differences occurred were identified by two-tailed t-tests. Latency period increased at all saccadic amplitudes at $1 \mathrm{hr}, 2 \mathrm{hrs}$ and $3 \mathrm{hrs}$, but the effect was not significant. However a significant effect can be seen only at $10^{\circ}(p=0.03)$ at $2 \mathrm{hrs}$ for rightward movements. No significant effect can be seen in leftward movements at all saccadic amplitudes. 


\subsubsection{Effect of Caffeine on Saccadic Eye Movements}

Results for the outcome measures are depicted in Table $3 \& 4$.

The results showed that caffeine decreased SD, increased PSV and LP compared to placebo for rightward and leftward eye movements.

\section{Saccadic duration:}

ANOVA with repeated measures showed significant effect of caffeine on saccadic duration for rightward movements at $30^{\circ}(p=0.02), 40^{\circ}(p=0.01), 50^{\circ}(p=0.001), 60^{\circ}(p=0.04)$. A similar effect was observed in case of leftward movements at $50^{\circ}(p=0.01), 60^{\circ}(p=0.05)$.

Compared to baseline a significant effect can be observed for rightward movements at $1 \mathrm{hr}$ at $30^{\circ}, 40^{\circ}, 50^{\circ}, 60^{\circ}$ and leftward movements at $40^{\circ}, 50^{\circ}$ and $60^{\circ}$ only.

Specific time points at which significant caffeine versus placebo differences occurred were identified by two-tailed paired t-tests. Saccadic duration differed significantly at $1 \mathrm{hr}$ only at $30^{\circ}(p=0.02), 40^{\circ}(p=0.03)$, $\left.50^{\circ}(p=0.02), 60^{\circ}(p=0.05)\right\}$ for rightward movements. No significant effect can be seen for leftward movements.

\section{Peak saccadic velocity:}

Caffeine increased the PSV for rightward movements significantly at $30^{\circ}(p=0.02), 40^{\circ}(p=0.01), 50^{\circ}(p<$ $0.0001), 60^{\circ}(\mathrm{P}=0.02)$. A significant effect was also observed for leftward movements at $40^{\circ}(p=0.04), 50^{\circ}(p=$ $0.03), 60^{\circ}(p=0.04)$.

Compared to baseline PSV increased significantly for rightward movements at $1 \mathrm{hr}$ only at $40^{\circ}, 50^{\circ}, 60^{\circ}$. A similar effect is also observed for leftward movements at $1 \mathrm{hr}$ at $40^{\circ}, 50^{\circ}, 60^{\circ}$. Specific time points at which significant caffeine versus placebo differences occurred were identified by two-tailed paired t-tests. PSV increased significantly at $1 \mathrm{hr}$ only $\left\{\right.$ at $\left.30^{\circ}(p=0.04), 40^{\circ}(p=0.01), 50^{\circ}(p=0.01), 60^{\circ}(p=0.05)\right\}$ for rightward movements. In contrast, no significant effect can be seen for leftward movements.

\section{Latency period:}

A significant decrease in latency period was observed for both rightward $(p=0.002)$ and leftward movements $(p=0.005)$ at $60^{\circ}$ only with repeated measures of ANOVA.

Compared to baseline, latency period decreased at all saccadic amplitude at $1 \mathrm{hr}$, however a significant effect can only be seen at $50^{\circ}$ and $60^{\circ}$ for rightward movements and at $60^{\circ}$ for leftward movements.

Specific time points at which significant caffeine versus placebo differences occurred were identified by two-tailed t-tests. Latency period decreased significantly at $1 \mathrm{hr}\left\{\right.$ at $\left.40^{\circ}(p=0.03), 50^{\circ}(p=0.01), 60^{\circ}(p=0.02)\right\}$ for rightward movements. Similarly a significant decrease in latency period was observed at $1 \mathrm{hr}$ at $\left\{\right.$ at $40^{\circ}(p=0.004)$, $\left.50^{\circ}(p=0.005), 60^{\circ}(p=0.03)\right\}$ for leftward movements.

\section{Discussion}

Eye movements are an integral and essential part of human foveated vision system and have seen massive advances during the last 50 yrs. A variety of experimental reliable methods for the measurement of saccadic eye movements involving human subjects for the evaluation of drug effects and in neurodegenerative diseases have been reported in medical literature.

Previous studies have attempted to characterize normal ranges for the saccadic duration, PSV and saccadic latency. But disagreement exists concerning the range of normal variability. In the present paper we standardized with two eye movement sequences and addressed the effect of zolpidem and caffeine on saccadic eye movements in a custom built device using forward sequence. The normative saccadic eye movement values, that were collected from 24 healthy subjects using both sequence was found to be reliable and accurate. There was no difference between genders on any of the saccadic parameters. As pointed out by Bolah, Sills, Kumley \& Honrubia, (1975), saccade duration increases approximately linearly with saccade amplitude, whereas PSV increases with amplitude at a decreasing rate. The values for SD, PSV and LP at various saccadic angles of both sequences, shown in the present study are in agreement with the previous literature (Wilson, Glue, Ball \& Nutt, 1993). The latency period of various amplitudes is not significantly different. As described by Hiroshi sasaki \& Niohiro ishii, (2005), the saccadic parameters of leftward movements are slower compared to rightward movements; this may be due to parallax between both the eyeballs. The present model was validated in a double blind three-way cross-over placebo controlled design using zolpidem $(5 \mathrm{mg})$ and caffeine $(500 \mathrm{mg})$ as standard drugs. Saccadic eye movements were recorded frequently to provide a measure of the time course of drug action. Peak effects were seen between 1-2 hrs for both drugs with the onset of action being $1 \mathrm{hrs}$ after drug intake. 
Compared to placebo, we found that acute administration of a low dose of zolpidem (5 mg) induces a significant decrease in PSV, increases saccadic latencies and produces sedation. The slowing of PSV by benzodiazepines is well established. The decrease in PSV and increase in latency period appears to be consistent with the results obtained in previous double-blind studies on zolpidem (Richens, Mercer, Jones, Griffiths \& Marshall, 1993) and other classes of sedative agents. Richens et al. (1993) showed lowering of PSV following single and repeated administration of $5 \mathrm{mg}, 10 \mathrm{mg}$ and $20 \mathrm{mg}$ zolpidem. A significant decrease in PSV following administration of $10 \mathrm{mg}$ diazepam, $20 \mathrm{mg}$ temazepam, an effect which persisted for $9 \mathrm{hrs}$ was reported by bittencourt et al (1981). Similarly Hopfenbeck, Cowley, Radant, Greenblatt \& Ray-Byrne, (1995) reported a decrease in PSV and increase in latency period following intravenous administration of diphenhydramine $(50 \mathrm{mg} / 70 \mathrm{~kg})$. Research by vander Meyden, Bartel, Sommers, Blom \& Pretorius, (1989) reported that $2 \mathrm{mg}$ clonazepam decreased PSV. These drug-induced changes in PSV and saccadic duration were due to a change in a local feedback loop that compares a non-visual eye position signal to the perceived target eccentricity (Jurgens, Becker \& Kornhuber, 1981).

Acute administration of caffeine showed a significant increase in PSV and decrease in saccadic duration and latency period. Though there is not much data on the effects of caffeine on saccadic eye movements, research by Smith, Brice, Nash, Rich \& Nutt (2003) showed a significant effect of caffeinated drink on saccadic eye movements. Similarly caffeine $200 \mathrm{mg}$ significantly reduced drowsiness and reaction times and these changes persisted for 3 to 4 hrs in healthy subjects in vigilance tests (Michael, Johns, Owen \& Patterson, 2008).

Tedeschi et al. (1983) showed that $15 \mathrm{mg}$ amphetamine sulphate decreased saccadic latency period on intravenous administration compared to placebo, however no significant effect on saccadic eye movements have been observed.

\section{Conclusion}

A noninvasive saccadic diagnostic system for recording saccadic eye movements in healthy human subjects has been developed indigenously. Saccadic eye movements in 24 normal subjects were found to be reliable and accurate. Saccadic eye movements are sensitive to a wide range of drugs and can be frequently and quickly measured without exhausting the subjects taking part, and once initiated, are beyond voluntary control provided that the angular movement does not exceed $60^{\circ}$. The present study describes a testing paradigm that is an acceptable and convenient method of evaluating drug effects in human subjects.

\section{Acknowledgements}

The authors have no conflict of interest to declare.

We are thankful to Indian Council of Medical Research, New Delhi, India for the financial support [Grant No: 44/7/2000/BMS] and facilities to carry out this study. We extend our thanks to Malini. V \& Satyanarayana. K, Dept. of Biomedical Engineering, Osmania university, Hyderabad, for helping us in designing the equipment and to Suraksha Pharmaceuticals Ltd, Roorkee, India, for providing study medication.

\section{References}

Abrams, R. A., Meyer, D. E., \& Kornblum, S. (1989). Speed and accuracy of saccadic eye movements: characteristics of impulse variability in the oculomotor system. Journal of Experimental Psychology: Human Perception and Performance, 15(3), 529-543. http://dx.doi.org/10.1037/0096-1523.15.3.529

Ball, D. M., Glue, P., Wilson, S., \& Nutt, D. J. (1991). Pharmacology of saccadic eye movements in man. 1. Effects of the benzodiazepine receptor ligands midazolam and flumazenil. Psychopharmacology (Berl), 105(3), 361-367. http://dx.doi.org/10.1007/BF02244431

Baloh, R. W., Sills, A. W., Kumley, W. E., \& Honrubia, V., (1975). Quantitative measurement of saccadic amplitude, duration, and velocity. Neurology, 25(11), 1065-1070.

Bittencourt, P. R., Wade, P., Smith, A. T., \& Richens, A. (1981). The relationship between peak velocity of saccadic eye movements and serum benzodiazepine concentrations. British journal of clinical pharmacology, 12(4), 523-533.

Ettinger, U., Kumari, V., Zachariah, E., Galea, A., Crawford, T. J., Corr, P. J., etal., (2003). Effects of Procyclidine on eye movements in schizophrenia. Neuropsychopharmacology, 28(12), 2199-2208.

Fletcher W. A., \& Sharpe J. A. (1986). Saccadic eye movement dysfunction in Alzheimer's disease. Ann Neurol, 20, 464-471. http://dx.doi.org/10.1002/ana.410200405

Fransson, P. A., Patel, M., Magnusson, M., Berg, S., Almbladh, P., \& Gomez, S. (2008). Effects of 24-hour and 
36-hour sleep deprivation on smooth pursuit and saccadic eye movements. Journal of Vestibular Research, $18(4), 209-222$.

Gao, F., Marshall, R. W., Sanders, L. D., \& Vickers, M. D. (1991). Effect of sub anaesthetic infusions of propofol on peak velocity of saccadic eye movement. European Journal of Anaesthesiology, 8(4), 267-276.

Hiroshi, Sasaki \& Naohiro, Ishii. (2005). A Study on Saccadic Eye Movements at the Mutual Gazing. GESTS International Transactions on Computer Science and Engineering, 18(1), 125-133.

Hopfenbeck, J. R., Cowley, D. S., Radant, A., Greenblatt, D. J., \& Roy-Byrne, P. P. (1995). Effects of diphenhydramine on human eye movements. Psychopharmacology (Berl), 118(3), 280-286. http://dx.doi.org/10.1007/BF02245956

Jurgens, R., Becker, W., \& Kornhuber, H. H., (1981). Natural and drug-induced variations of velocity and duration of humans saccadic eye movements: evidence for a control of the neural pulse generator. Biological Cybernetics, 39(2), 87-96. http://dx.doi.org/10.1007/BF00336734

Khan, O., Taylor, S. J., Jones, J.G., Swart, M., Hanes, D. P., \&. Carpenter, R. H. S. (1999). Effects of low-dose isoflurane on saccadic eye movement generation. Anaesthesia, 54(2), 142-145. http://dx.doi.org/10.1046/j.1365-2044.1999.00701.x

Kirchner, H., \& Thorpe S. J. (2006). Ultra-rapid object detection with saccadic eye movements: Visual $\begin{array}{lllll}\text { processing } & \text { speed }\end{array}$ http://dx.doi.org/10.1016/j.visres.2005.10.002

Michael, N., Johns, M., Owen, C., \& Patterson, J. (2008). Effects of caffeine on alertness as measured by infrared reflectance oculography. Psychopharmacology (Berl), 200(2), 255-260. http://dx.doi.org/10.1007/s00213-008-1202-z

Richens, A., Mercer, A. J., Jones, D. M., Griffiths, A. \& Marshall, R.W. (1993). Effects of zolpidem on saccadic eye movements and psychomotor performance: a double-blind, placebo controlled study in healthy volunteers. British Journal of Clinical Pharmacology, 36(1), 61-65.

Smith, A., Brice, C., Nash, J., Rich, N., \& Nutt, D. J. (2003). Caffeine and central noradrenaline: effects on mood, cognitive performance, eye movements and cardiovascular function. Journal of psychopharmacology, 17(3), 283-292. http://dx.doi.org/10.1177/02698811030173010

Tedeschi, G., Bittencourt, P. R., Smith, A. T., \& Richens, A. (1983). Effect of amphetamine on saccadic and smooth pursuit eye movements. Psychopharmacology, 79(2-3), 190-192. http://dx.doi.org/10.1007/BF00427810

Van der Meyden, C. H., Bartel, P. R., Sommers, D. K., Blom, M., \& Pretorius, L. C. (1989). Effects of clobazam and clonazepam on saccadic eye movements and other parameters of psychomotor performance. European Journal of Clinical Pharmacology, 37(4), 365-369. http://dx.doi.org/10.1007/BF00558501

Vidapanakanti, M., Kakarla, S., Katukojwala, S., \& Naidu, M. U. R. (2009). Analysis of Saccadic Eye movements of Epileptic Patients Using Indigenously Designed and Developed Saccadic Diagnostic System. IFMBE Proceedings, 2 (1), 431-434. http://dx.doi.org/10.1007/978-3-540-92841-6_106

Wilson, S. J., Glue, P., Ball, D., \& Nutt, D. J. (1993). Saccadic eye movement parameters in normal subjects. $\begin{array}{llll}\text { Electroencephalography and clinical neurophysiology, } & 86(1), & 69-74 .\end{array}$ http://dx.doi.org/10.1016/0013-4694(93)90068-7

Yoshizumi, J., Marshall, R. W., \& Vickers, M. D. (1991). Effect of low concentrations of cyclopropane and halothane on peak velocity of saccadic eye movement. British Journal of Anaesthesia, 67(6), 735-740. http://dx.doi.org/10.1093/bja/67.6.735

Zils, E., Sprenger, A., Heide, W., Born, J., \& Gais, S. (2005). Differential effects of sleep deprivation on saccadic eye movements. Sleep, 28(9), 1109-1115. 